\title{
Oscillations of a Class of Forced Second-Order Differential Equations with Possible Discontinuous Coefficients
}

\author{
Siniša Miličić, Mervan Pašić, and Darko Žubrinić \\ Department of Mathematics, Faculty of Electrical Engeneering and Computing, University of Zagreb, 10000 Zagreb, Croatia \\ Correspondence should be addressed to Mervan Pašić; mervan.pasic@gmail.com
}

Received 29 January 2013; Accepted 16 April 2013

Academic Editor: Ondrej Dosly

Copyright () 2013 Siniša Miličić et al. This is an open access article distributed under the Creative Commons Attribution License, which permits unrestricted use, distribution, and reproduction in any medium, provided the original work is properly cited.

We study the oscillation of all solutions of a general class of forced second-order differential equations, where their second derivative is not necessarily a continuous function and the coefficients of the main equation may be discontinuous. Our main results are not included in the previously published known oscillation criteria of interval type. Many examples and consequences are presented illustrating the main results.

\section{Introduction}

Let $t_{0}>0$ and let $A C_{\text {loc }}\left(\left[t_{0}, \infty\right), \mathbb{R}\right)$ denote the set of all real functions absolutely continuous on every bounded interval $[a, b] \subset\left[t_{0}, \infty\right)$. We study the oscillatory behaviour of all solutions $x=x(t)$ of the following class of forced secondorder differential equations:

$$
\begin{gathered}
\left(r(t) \Phi\left(x(t), x^{\prime}(t)\right)\right)^{\prime}+q(t) f(x(t))=e(t), \\
\text { a.e. in }\left[t_{0}, \infty\right), \\
x, r \Phi\left(x, x^{\prime}\right) \in A C_{\mathrm{loc}}\left(\left[t_{0}, \infty\right), \mathbb{R}\right),
\end{gathered}
$$

where the functions $\Phi: \mathbb{R}^{2} \rightarrow \mathbb{R}, \Phi=\Phi(u, v), f:$ $\mathbb{R} \rightarrow \mathbb{R}$, and $f=f(u)$ satisfy some general conditions given in Section 2. A continuous function $x=x(t)$ is said to be oscillatory if there is a sequence $t_{n} \in\left[t_{0}, \infty\right)$, such that $x\left(t_{n}\right)=0$ for all $n \in \mathbb{N}$ and $t_{n} \rightarrow \infty$ as $n \rightarrow \infty$. A differential equation is oscillatory if all its solutions are oscillatory.

The forcing term $e(t)$ is a sign-changing function (possibly discontinuous). This can be formulated by the following hypothesis: for every $T \geq t_{0}$ there exist two intervals $\left(a_{1}, b_{1}\right)$ and $\left(a_{2}, b_{2}\right), T \leq a_{1}<b_{1} \leq a_{2}<b_{2}$, such that

$$
\begin{aligned}
& e(t) \geq 0, \quad t \in\left(a_{1}, b_{1}\right), \\
& e(t) \leq 0, \quad t \in\left(a_{2}, b_{2}\right) .
\end{aligned}
$$

The coefficient $q(t)$ may be a discontinuous function on $\left[t_{0}, \infty\right)$ and the case $x \notin C^{2}\left(\left(t_{0}, \infty\right), \mathbb{R}\right)$ occurs in our main results and examples too. Two important classes of functions $\Phi(u, v)$ are included in the differential operator $\left(r(t) \Phi\left(x, x^{\prime}\right)\right)^{\prime}$ as

$$
\Phi(u, v)=\phi(u) v, \quad \Phi(u, v)=\frac{\phi(u) v}{\sqrt{1+v^{2}}}, \quad(u, v) \in \mathbb{R}^{2}
$$

The first one is the classic second-order differential operator which is linear in $x^{\prime}$ and the second one is the so-called one-dimensional mean curvature differential operator; see Examples 1 and 2.

Depending on $q(t)$, we propose the following four simple models for (1):

(i) $q(t)$ is strictly positive and continuous on $\left[t_{0}, \infty\right)$ as

$$
\begin{aligned}
& x^{\prime \prime}+4 m^{2} f(x)=h(\sin (m t)), \quad \text { a.e. in }\left[t_{0}, \infty\right), \\
& x, x^{\prime} \in A C_{\text {loc }}\left(\left[t_{0}, \infty\right), \mathbb{R}\right), \quad x \notin C^{2}\left(\left(t_{0}, \infty\right), \mathbb{R}\right) ;
\end{aligned}
$$

(ii) $q(t)$ is nonnegative and continuous on $\left[t_{0}, \infty\right)$ as

$$
\begin{array}{r}
x^{\prime \prime}+m^{2} \pi^{2}[\cos (m t)]^{+} f(x)=h(\sin (m t)), \\
\text { a.e. in }\left[t_{0}, \infty\right), \\
x, x^{\prime} \in A C_{\mathrm{loc}}\left(\left[t_{0}, \infty\right), \mathbb{R}\right) ;
\end{array}
$$




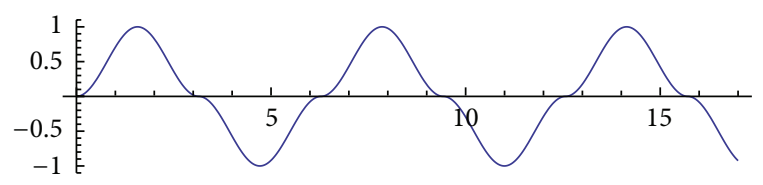

Figure 1: Function $x(t)=|\sin (t)| \sin (t)$ is a solution of (4).

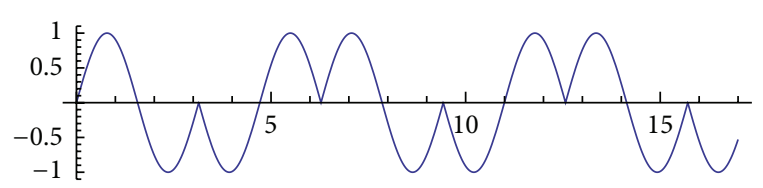

FIGURE 2: $x^{\prime}(t)=2|\sin (t)| \cos (t)$ and hence $x^{\prime \prime}(t)$ is not a continuous function.

(iii) $q(t)$ is nonnegative and discontinuous on $\left[t_{0}, \infty\right)$ as

$$
\begin{gathered}
x^{\prime \prime}+4 m^{2}[\operatorname{sign}(\cos (m t))]^{+} f(x)=h(\sin (m t)), \\
\text { a.e. in }\left[t_{0}, \infty\right), \\
x, x^{\prime} \in A C_{\text {loc }}\left(\left[t_{0}, \infty\right), \mathbb{R}\right) ;
\end{gathered}
$$

(iv) $q(t)$ is sign changing and discontinuous on $\left[t_{0}, \infty\right)$ as

$$
\begin{gathered}
x^{\prime \prime}+4 m^{2} \operatorname{sign}(\cos (m t)) f(x)=h(\sin (m t)), \\
\text { a.e. in }\left[t_{0}, \infty\right), \\
x, x^{\prime} \in A C_{\mathrm{loc}}\left(\left[t_{0}, \infty\right), \mathbb{R}\right),
\end{gathered}
$$

where $m \in \mathbb{N}$ and $h(s)$ is an arbitrary function such that $h(s) s>0$ for all $s \neq 0$, for instance, $h(s)=s$ or $h(s)=\operatorname{sign}(s)$. According to Corollaries 7 and 10, we will show that (4)(7) are oscillatory provided the function $f=f(u)$ satisfies $f(u) / u \geq K \geq 1$ for all $u \neq 0$; see Examples $8-13$. It is interesting that in particular for $f(u)=u$ and $h(s)=$ $2 m^{2} \operatorname{sign}(s),(4)$ allows an explicit oscillatory solution $x(t)=$ $|\sin (m t)| \sin (m t)$ as shown in Figures 1 and 2.

Moreover, as a consequence of Corollary 7, one can show that all solutions of (4) are oscillatory; for details see Example 8. The main goal of this paper is to give some sufficient conditions on functions $\Phi(u, v), f(u)$ and the coefficients $r(t), q(t)$, and $e(t)$ such that (1) is oscillatory; see Theorems 3 and 4 . It will also cover the model equations (4)(7) as well as some other examples presented in Section 2.

To the best of our knowledge, it seems that there are only few papers which study the oscillation of the second-order differential equations with nonsmooth (local integrable) coefficients; see [1-3]. More precisely, in [1] the author studied the interval oscillation criteria for the following second-order half-linear differential equation:

$$
\begin{gathered}
\left(r(t)\left|x^{\prime}(t)\right|^{\sigma-1} x^{\prime}(t)\right)^{\prime}+q(t)|x(t)|^{\sigma-1} x(t)=0, \\
\text { a.e. in }(0, \infty), \\
x, r\left|x^{\prime}\right|^{\sigma-1} x^{\prime} \in A C_{\mathrm{loc}}((0, \infty), \mathbb{R}),
\end{gathered}
$$

where $\sigma>1$ and $1 / r, q \in L_{\text {loc }}((0, \infty), \mathbb{R})$ such that $\int_{0}^{\infty} r^{-1 / \sigma}(t) d r=\infty$. See also [2] but with the solution space $C^{1}((0, \infty), \mathbb{R})$ instead of $A C_{\text {loc }}((0, \infty), \mathbb{R})$, that is, $x$ and $r\left|x^{\prime}\right|^{\sigma-1} x^{\prime} \in C^{1}((0, \infty), \mathbb{R})$.

In [3], the authors consider the following second-order differential equation:

$$
\left(r(t) x^{\prime}(t)\right)^{\prime}+Q\left(t, x(t), x^{\prime}(t)\right)=0, \quad t \geq t_{0}
$$

where $r(t)>0$ a.e. in $\left[t_{0}, \infty\right), 1 / r \in L_{\text {loc }}\left(\left[t_{0}, \infty\right), \mathbb{R}\right)$, and $\mathrm{Q}(t, y, z)$ is locally integrable function in $t$ and continuous in $(y, z)$. Equation (9) allows the forcing term $e(t)$ in the next sense as follows:

$$
\begin{array}{r}
y Q(t, y, z) \geq q(t) y f(y)-e(t) y \\
\forall(t, y, z) \in\left[t_{0}, \infty\right) \times \mathbb{R}^{2},
\end{array}
$$

where $e=e(t)$ satisfies (2), but the functions $q=q(t)$ and $f=f(y)$ are smooth enough in their variables, that is, $q \in$ $C\left(\left[t_{0}, \infty\right), \mathbb{R}\right)$ and $f \in C^{1}(\mathbb{R}, \mathbb{R})$.

On certain oscillation criteria for various classes of forced second-order differential equations with continuous coefficients, we refer the reader to [4-13]. Our method modifies a recently used one in $[14,15]$ and it contains the classic Riccati transformation of the main equation, a blow-up argument and pointwise comparison principle. The comparison principle applies to all sub-and supersolutions of a class of the generalized Riccati differential equations with nonlinear terms that are supposed to be locally integrable in the first variable and locally Lipschitz continuous in the second variable.

\section{Hypotheses, Results, and Consequences}

First of all, the function $\Phi(u, v)$ which appears in the secondorder differential operator of (1) satisfies

$$
|u|^{\gamma-2} v \Phi(u, v) \geq g(|\Phi(u, v)|) \quad \forall u, v \in \mathbb{R}
$$

where $\gamma \geq 2$ and $g: \mathbb{R}_{+} \rightarrow \mathbb{R}_{+}$is a locally Lipschitz function $g_{0}: \mathbb{R}_{+} \rightarrow \mathbb{R}_{+}$satisfying

$$
\begin{gathered}
g(c s) \geq c^{\gamma} g_{0}(s) \quad \forall c>0, s>0 \\
g_{0}(s)+M_{0} \geq s^{2} \quad \text { for some } M_{0} \geq 0 \text { and all } s \in \mathbb{R}_{+} .
\end{gathered}
$$

In most cases, $g(s)=g_{0}(s) g_{1}(s), s>0$, where $g_{0}(s)=s^{\gamma}$, $\gamma \geq 2$, and $g_{1}(s)$ is an arbitrary function satisfying $g_{1}(s) \geq 1$. Thus, for such $g(s)$ with $g_{1}(s) \equiv 1$, condition (11) became:

$$
|u|^{\gamma-2} v \Phi(u, v) \geq|\Phi(u, v)|^{\gamma} \quad \forall u, v \in \mathbb{R} .
$$

It is not difficult to check that if $g_{0} \in C^{1}\left(\mathbb{R}_{+}\right)$or $g_{0}(s)$ is a convex function, then it is locally Lipschitz on $\mathbb{R}_{+}$too; see for instance [16, Theorem 1.3.3].

Two essential classes of the second-order differential operators $\left(r(t) \Phi\left(x, x^{\prime}\right)\right)^{\prime}$ satisfy condition (13), as is shown in the next examples. 
Example 1. We consider the second-order differential operator which is linear in $x^{\prime}$ as follows:

$$
\left(r(t) \Phi\left(x, x^{\prime}\right)\right)^{\prime}=\alpha\left(\phi(x) x^{\prime}\right)^{\prime}
$$

where $\alpha>0$ and $0 \leq \phi(u) \leq 1$ for all $u \in \mathbb{R}$. Obviously, the function $\Phi(u, v)=\phi(u) v$ satisfies condition (13) in particular for $\gamma=2$. Two usual choices for $\phi(u)$ are $\phi(u)=|\sin u|$ and $\phi(u)=|u| /(1+|u|)$.

Example 2. We consider a quasilinear differential operator (the so-called one-dimensional prescribed mean curvature operator) as follows:

$$
\left(r(t) \Phi\left(x, x^{\prime}\right)\right)^{\prime}=\alpha\left(\phi(x) \frac{x^{\prime}}{\sqrt{1+x^{\prime 2}}}\right)^{\prime}
$$

where $\alpha>0$ and $0 \leq \phi^{\gamma-1}(u) \leq|u|^{\gamma-2}$ for all $u \in \mathbb{R}$. It is not difficult to check that condition (13) is satisfied in particular for $\Phi(u, v)=\phi(u) v /\left(1+v^{2}\right)^{1 / 2}$ and for any $\gamma>1$. For $\phi(u)$, we can take the same choice as in the previous example.

Next, we suppose the existence of a constant $K$ such that

$$
\frac{f(u)}{u} \geq K>0 \quad \forall u \neq 0 .
$$

In order to simplify our consideration here, in many examples we often use $f(u)=K u$.

Condition (2) means that there exists a sequence of pairs of intervals $J_{1 j}=\left[a_{1 j}, b_{1 j}\right]$ and $J_{2 j}=\left[a_{2 j}, b_{2 j}\right], j \in \mathbb{N}$, contained in $\left(t_{0}, \infty\right)$, such that the sequences $\left(a_{1 j}\right)_{j \geq 1},\left(b_{1 j}\right)_{j \geq 1}$, $\left(a_{2 j}\right)_{j \geq 1}$, and $\left(b_{2 j}\right)_{j \geq 1}$ are increasing, $a_{1 j}<b_{1 j} \leq a_{2 j}<b_{2 j}$ for each $j$, and

$$
\begin{gathered}
e(t) \geq 0 \quad \text { on } J_{1 j}, \quad e(t) \leq 0 \text { on } J_{2 j} \\
\text { for each } j \in \mathbb{N}, \\
\lim _{j \rightarrow \infty} a_{1 j}=\infty .
\end{gathered}
$$

On the intervals $J_{1 j}$ and $J_{2 j}$, the coefficient $r(t)$ satisfies

$$
\begin{array}{r}
r(t)>0 \quad \text { on } J_{i j}, r^{1-\gamma} \in L^{1}\left(J_{i j}\right) \\
\forall i \in\{1,2\}, j \in \mathbb{N} .
\end{array}
$$

Let there be a real function $C=C(t), C \in$ $L_{\text {loc }}^{1}\left(\left(t_{0}, \infty\right), \mathbb{R}\right)$, and let there exist a sequence of positive real numbers $\left(\lambda_{j}\right)_{j \in \mathbb{N}}$ such that

$$
\begin{array}{r}
C(t) \geq 0 \quad \text { on } J_{i j}, c_{i j}:=\int_{J_{i j}} C(\tau) d \tau>0 \\
\forall i \in\{1,2\}, j \in \mathbb{N}, \\
\frac{1}{c_{i j}} C(t) \leq \frac{1}{\pi} \min \left\{\left(\lambda_{j} r(t)\right)^{1-\gamma}, \frac{K}{M_{0}+1} \lambda_{j} q(t)\right\} \\
\forall t \in J_{i j}, i \in\{1,2\}, j \in \mathbb{N},
\end{array}
$$

where $\gamma, M_{0}$, and $K$ are constants defined in (11), (12), and (16), respectively.

The proof of the following main result will be presented in Section 4.

Theorem 3. Let the functions $\Phi(u, v), f(u), e(t)$, and $r(t)$ satisfy (11), (12), (16), (17), and (18), respectively. Let $q(t) \geq 0$ and $q(t) \neq 0$ on each interval $J_{i j}, i \in\{1,2\}, j \in \mathbb{N}$. If (19) is fulfilled, then (1) is oscillatory.

Condition (19) can be replaced by an equivalent one, which has a more practical value and takes a simpler form since we do not need a sequence of auxiliary parameters $\left(\lambda_{j}\right)_{j \in \mathbb{N}}$ : let there be a real function $C=C(t), C \in$ $L_{\text {loc }}^{1}\left(\left(t_{0}, \infty\right), \mathbb{R}\right)$ such that

$$
\begin{array}{r}
C(t) \geq 0 \quad \text { on } J_{i j}, c_{i j}:=\int_{I_{i j}} C(\tau) d \tau>0 \quad \forall i \in\{1,2\}, \\
j \in \mathbb{N}, \\
\sup _{t \in I_{i j}}\left[\frac{C(t)}{q(t)}\right] \sup _{t \in I_{i j}}\left[r(t) C(t)^{1 /(\gamma-1)}\right] \leq \frac{K}{M_{0}+1}\left(\frac{c_{i j}}{\pi}\right)^{\gamma /(\gamma-1)} \\
\forall i \in\{1,2\}, j \in \mathbb{N},
\end{array}
$$

where $\gamma, M_{0}$, and $K$ are constants defined in (11), (12), and (16), respectively. Since we will show that (19) and (20) are equivalent, see page 8 , the next oscillation criterion immediately follows from Theorem 3.

Theorem 4. Let the functions $\Phi(u, v), f(u), e(t)$, and $r(t)$ satisfy (11), (12), (16), (17), and (18), respectively. Let $q(t) \geq 0$ and $q(t) \neq \equiv$ on each interval $J_{i j}, i \in\{1,2\}, j \in \mathbb{N}$. If $(20)$ is fulfilled, then (1) is oscillatory.

Remark 5. Assuming that $L:=\lim _{j \rightarrow \infty} a_{1 j}<\infty$, we can ensure the oscillation in the point $L$. Note that $L=$ $\lim _{j \rightarrow \infty} a_{2 j}$ since $a_{1 j}<a_{2 j}<a_{1 j+1}$. Thus, we can generate a one-sided (right) limit.

Now, we consider some consequences of Theorem 4, which depend on the qualitative properties of the coefficient $q(t)$.

Substituting $C(t) \equiv 1$ in (20), Theorem 4 implies the following result involving lower bounds on the lengths of intervals $\left|J_{i j}\right|=b_{i j}-a_{i j}$.

Corollary $6(q(t)$ is positive). Let the functions $\Phi(u, v), f(u)$, $e(t)$, and $r(t)$ satisfy (11), (12), (16), (17), and (18), respectively. Let $\inf _{t \in J_{i j}} q(t)>0$ for each $i \in\{1,2\}, j \in \mathbb{N}$. If

$$
\left|J_{i j}\right| \geq \pi\left(\frac{\left(M_{0}+1\right) \sup _{t \in J_{i j}} r(t)}{\operatorname{Kinf}_{t \in J_{i j}} q(t)}\right)^{(\gamma-1) / \gamma} \quad \forall i \in\{1,2\}, j \in \mathbb{N}
$$

then (1) is oscillatory. 
Corollary $7(q(t)$ is bounded from below by a positive constant). Let the functions $\Phi(u, v), f(u), e(t)$, and $r(t)$ satisfy (11), (12), (16), (17), and (18), respectively. Let there be two constants $r_{0}, q_{0}$ satisfying

$$
0<r(t) \leq r_{0}, \quad q(t) \geq q_{0}>0 \quad \forall i \in\{1,2\}, j \in \mathbb{N} .
$$

If

$$
\left|J_{i j}\right| \geq \pi\left(\frac{\left(M_{0}+1\right) r_{0}}{K q_{0}}\right)^{(\gamma-1) / \gamma} \quad \forall i \in\{1,2\}, j \in \mathbb{N},
$$

then (1) is oscillatory, where $\gamma, M_{0}$, and $K$ are constants defined in (11), (12), and (16), respectively.

Example 8 (oscillation of (4)). We know that $x(t)=$ $|\sin (m t)| \sin (m t)$ is an oscillatory solution of (4). However, according to Corollary 7, we can show that all solutions of (4) are oscillatory. Indeed, since $\Phi(u, v) \equiv v$, the conditions (11) and (12) are satisfied especially for $\gamma=2, g(s)=g_{0}(s)=s^{2}$ and $M_{0}=0$. Next, $f(u) \equiv u$ implies that condition (16) is satisfied especially for $K=1$. Since $r(t) \equiv 1$ and $q(t) \equiv 4 \mathrm{~m}^{2}$, it is clear that conditions (18) and (22) are also satisfied in particular for $r_{0}=1$ and $q_{0}=4 \mathrm{~m}^{2}$. Moreover, since $e(t)=$ $h(\sin (m t))$ and $h(s) s>0, s \neq 0$, we have that (17) is fulfilled for $a_{1 j}=2 j \pi / m, b_{1 j}=(2 j+1) \pi / m=a_{2 j}$ and $b_{2 j}=(2 j+2) \pi / m$. Moreover,

$$
\left|J_{i j}\right|=b_{i}-a_{i}=\frac{\pi}{m}>0, \quad i \in\{1,2\} .
$$

Hence, we conclude that the required condition (23) is fulfilled, that is,

$$
\begin{aligned}
\left|J_{i j}\right| & =\frac{\pi}{m} \geq \frac{\pi}{\sqrt{4 m^{2}}} \\
& =\pi\left(\frac{\left(M_{0}+1\right) r_{0}}{q_{0}}\right)^{(\gamma-1) / \gamma} \geq \pi\left(\frac{\left(M_{0}+1\right) r_{0}}{K q_{0}}\right)^{(\gamma-1) / \gamma} .
\end{aligned}
$$

Thus, all conditions of Corollary 7 are satisfied and hence (4) is oscillatory.

Example 9. We consider the following class of equations:

$$
\begin{gathered}
x^{\prime \prime}-2 \frac{S^{\prime \prime}(t)}{S(t)} x=2 \operatorname{sign}(S(t)) S^{\prime 2}(t), \quad \text { a.e. in }\left[t_{0}, \infty\right), \\
x, x^{\prime} \in A C_{\mathrm{loc}}\left(\left[t_{0}, \infty\right), \mathbb{R}\right), \quad x \notin C^{2}\left(\left(t_{0}, \infty\right), \mathbb{R}\right),
\end{gathered}
$$

where $S=S(t), S \in C^{2}(\mathbb{R})$ is an oscillatory function such that the zeros $t_{n}$ of the function $\operatorname{sign}(S(t)) S^{\prime 2}(t)$ satisfy $t_{n} \rightarrow \infty$, there is a $\tau_{0} \in \mathbb{R}$ such that $t_{n+1}-t_{n} \geq \tau_{0}>0$ for all $n \in \mathbb{N}$, and $S(t) \neq 0$ on $\left(t_{n}, t_{n+1}\right)$. This equation allows an explicitly given oscillatory solution $x(t)=|S(t)| S(t)$. Moreover, if there is a constant $s_{0}>0$ such that

$$
-2 \frac{S^{\prime \prime}(t)}{S(t)} \geq s_{0}, \quad t \in\left(t_{n}, t_{n+1}\right), \quad \tau_{0} \geq \frac{\pi}{\sqrt{s_{0}}},
$$

then by Corollary 7 we conclude that (26) is oscillatory. Indeed, conditions (11), (12), and (16) are satisfied by the same reasons as in Example 8. Condition (18) is satisfied because of $r(t) \equiv 1$. Also, from (27) it follows that (22) and (23) are fulfilled in particular for $\gamma=2, M_{0}=0, r_{0}=1, q_{0}=s_{0}$, and $K \geq 1$, that is,

$$
\begin{aligned}
\left|J_{i j}\right| & =\left|t_{j+1}-t_{j}\right| \geq \tau_{0} \geq \frac{\pi}{\sqrt{s_{0}}} \\
& =\pi\left(\frac{\left(M_{0}+1\right) r_{0}}{q_{0}}\right)^{(\gamma-1) / \gamma} \geq \pi\left(\frac{\left(M_{0}+1\right) r_{0}}{K q_{0}}\right)^{(\gamma-1) / \gamma} .
\end{aligned}
$$

Hence Corollary 7 proves this result.

As the second consequence of Theorem 3 is unlike the first one, we consider the case when the coefficient $q(t)$ is not a strictly positive function. Here by $\{q=0\}$ we denote the set of all $t \in \mathbb{R}$ such that $q(t)=0$.

Corollary $10(q(t)$ is nonnegative, but not $\equiv 0)$. Let the functions $\Phi(u, v), f(u), e(t)$, and $r(t)$ satisfy (11), (12), (16), (17), and (18), respectively. Let $q(t) \geq 0$ on each interval $J_{i j}$, $i \in\{1,2\}, j \in \mathbb{N}$, such that

$$
q_{i j}:=\int_{J_{i j}} q(\tau) d \tau>0, \quad i \in\{1,2\}, j \in \mathbb{N} .
$$

If

$$
\begin{aligned}
& \frac{q_{i j}^{\gamma}}{q(t)} \geq \pi^{\gamma}\left(\frac{\left(M_{0}+1\right) r(t)}{K}\right)^{\gamma-1}, \\
& t \in J_{i j} \backslash\{q=0\}, i \in\{1,2\}, j \in \mathbb{N},
\end{aligned}
$$

then (1) is oscillatory.

Proof. It suffices to show that (30) is equivalent to the existence of a real number $\lambda_{j}$ such that

$$
\begin{array}{r}
\lambda_{j} \geq \frac{\pi\left(M_{0}+1\right)}{K} \frac{1}{q_{i j}}, \quad \frac{1}{q_{i j}} q(t) \leq \frac{1}{\pi}\left(\lambda_{j} r(t)\right)^{1-\gamma}, \\
t \in J_{i j}, \quad i \in\{1,2\}, j \in \mathbb{N} .
\end{array}
$$

The claim will then follow from Theorem 3. Inequality (31) is for any $t \in J_{i j} \backslash\{q=0\}$ equivalent to

$$
\frac{\pi\left(M_{0}+1\right)}{K} \frac{1}{q_{i j}} \leq \lambda_{j} \leq\left(\frac{q_{i j}}{\pi q(t)}\right)^{1 /(\gamma-1)} \frac{1}{r(t)},
$$

that is, to

$$
\frac{\pi\left(M_{0}+1\right)}{K} \frac{1}{q_{i j}} \leq\left(\frac{q_{i j}}{\pi q(t)}\right)^{1 /(\gamma-1)} \frac{1}{r(t)} .
$$

This inequality is easily seen to be equivalent to (30) for any $t \in J_{i j} \backslash\{q=0\}$. Note that if $t \in\{q=0\}$, then the second inequality in (31) is trivially satisfied. 
The following three examples are simple consequences of Corollary 10.

Example 11 (oscillation of (5)). Equation (5) as well as equation

$$
\begin{gathered}
x^{\prime \prime}+m^{2} \pi^{2}[\sin (m t)]^{+} f(x)=h(\cos (m t)), \\
\text { a.e. in }\left[t_{0}, \infty\right), \\
x, x^{\prime} \in A C_{\text {loc }}\left(\left[t_{0}, \infty\right), \mathbb{R}\right),
\end{gathered}
$$

are oscillatory, where $f(u)$ satisfies (16) with $K \geq 1$ and $h(s) s>0, s \neq 0$. Indeed, in (5) we have $r(t) \equiv 1, q(t)=$ $m^{2} \pi^{2}[\cos (m t)]$ and $e(t)=h(\sin (m t))$, and so, (17) and (18) are fulfilled, and $q(t) \geq 0, q(t) \not \equiv 0$ on each interval $J_{i j}, i \in\{1,2\}$, $j \in \mathbb{N}$, where $J_{1 j}=\left[a_{1 j}, b_{1 j}\right], J_{2 j}=\left[a_{2 j}, b_{2 j}\right]$, and

$$
\begin{gathered}
a_{1 j}=\frac{2 j \pi}{m}, \quad b_{1 j}=\frac{(2 j+1 / 2) \pi}{m}, \\
a_{2 j}=\frac{(2 j+3 / 2) \pi}{m}, \quad b_{2 j}=\frac{(2 j+2) \pi}{m} .
\end{gathered}
$$

Moreover,

$$
\begin{aligned}
q_{i j} & =\int_{J_{i j}} q(\tau) d \tau \\
& =m^{2} \pi^{2} \int_{a_{i j}}^{b_{i j}}[\cos (m \tau)]^{+} d \tau=m \pi^{2}, \quad i \in\{1,2\}, j \in \mathbb{N},
\end{aligned}
$$

and since $\gamma=2, r(t) \equiv 1, K \geq 1$, and $M_{0}=0$, for $\lambda_{j}=1 /(m \pi)$ we have

$$
\begin{aligned}
& \lambda_{j}=\frac{1}{m \pi} \geq \frac{1}{K} \frac{1}{m \pi}=\frac{\pi\left(M_{0}+1\right)}{K} \frac{1}{m \pi^{2}} \\
&=\frac{\pi\left(M_{0}+1\right)}{K} \frac{1}{q_{i j}}, \quad i \in\{1,2\}, j \in \mathbb{N}, \\
& \frac{1}{q_{i j}} q(t)=\frac{1}{m \pi^{2}} m^{2} \pi^{2}[\cos (m t)]^{+} \leq m \\
&=\frac{1}{\pi} \frac{1}{(1 / m \pi)}=\frac{1}{\pi} \frac{1}{\left(\lambda_{j} r(t)\right)^{\gamma-1}}, \\
& t \in J_{i j}, i \in\{1,2\}, j \in \mathbb{N} .
\end{aligned}
$$

Thus, the required condition (30) is fulfilled in this case and hence we may apply Corollary 10 to (5) to verify that this equation is oscillatory. Analogously, we can show that (34) is oscillatory too.

In the next example, the coefficient $q(t)$ is a discontinuous function on $\left[t_{0}, \infty\right)$.

Example 12 (oscillation of (6)). If $f(u)$ satisfies (16) with $K \geq$ 1 , then (6) is oscillatory since the required condition (30) is satisfied especially for $q(t)=4 m^{2} \operatorname{sign}(\cos (m t))$ on $J_{i j}, \lambda_{j}=$ $1 /(2 m)$, and $J_{i j}$ as in (35), $\gamma=2, r(t) \equiv 1, K \geq 1$, and $M_{0}=0$.
Indeed

$$
\begin{aligned}
& q_{i j}=\int_{J_{i j}} q(\tau) d \tau=4 m^{2} \int_{J_{i j}}[\operatorname{sign}(\cos (m \tau))]^{+} d \tau \\
&=4 m^{2} \frac{\pi}{2 m}=2 m \pi, \quad i \in\{1,2\}, j \in \mathbb{N}, \\
& \lambda_{j}=\frac{1}{2 m}=\frac{\pi}{2 m \pi}=\pi\left(M_{0}+1\right) \frac{1}{q_{i j}} \\
& \geq \frac{\pi\left(M_{0}+1\right)}{K} \frac{1}{q_{i j}}, \quad i \in\{1,2\}, j \in \mathbb{N}, \\
& \frac{1}{q_{i}} q(t)=\frac{1}{2 m \pi} 4 m^{2}[\operatorname{sign}(\cos (m t))]^{+} \\
& \leq \frac{2 m}{\pi}=\frac{1}{\pi} \frac{1}{\sqrt{1 /\left(4 m^{2}\right)}}=\frac{1}{\pi}\left(\lambda_{j} r(t)\right)^{\gamma-1}, \\
& t \in J_{i j}, \quad i \in\{1,2\}, j \in \mathbb{N},
\end{aligned}
$$

which shows that (30) is satisfied.

At the end of this section, we consider the case when $q(t)$ changes sign on $\left[t_{0}, \infty\right)$ but with the help of Corollary 10 since $q(t)$ is strictly positive on all intervals $J_{i j}$.

Example 13 (oscillation of (7)). If $f(u)$ satisfies (16) with $K \geq$ 1 , then model equation (7) is oscillatory. In fact, let $J_{i j}$ be as in (35). If $h(s) s>0, s \neq 0$, it is clear that $e(t)=h(\sin (m t))$ satisfies (2), that is, $e(t) \geq 0$ on $J_{1 j}$ and $e(t) \leq 0$ on $J_{2 j}$. On the other hand, we have $q(t)=4 m^{2} \operatorname{sign}(\cos (m t))$ on $J_{i j}$ and so $q(t) \geq 0, q(t) \not \equiv 0$ on $J_{i j}$ for all $i \in\{1,2\}, j \in \mathbb{N}$. Hence, the proof of the fact that all assumptions of Corollary 7 are fulfilled is the same as in the preceding example.

\section{Proofs of Theorems 3 and 4}

Let $A C_{\text {loc }}([a, b), \mathbb{R})$ denote the set of all real functions which are absolutely continuous on every interval $[a, b-\varepsilon]$, where $\varepsilon \in(0, b-a)$. For arbitrary numbers $T_{0}<T^{*}$ and functions $G=G(t, u)$ and $b=b(t)$, we consider the ordinary differential equation

$$
w^{\prime}=G(t, w)+b(t), \quad \text { a.e. in }\left[T_{0}, T^{*}\right),
$$

which generalizes the classic Riccati equation $w^{\prime}=a(t) w^{2}+$ $b(t)$, where $a(t)$ is an arbitrary function. We associate to (39) the corresponding sub- and supersolutions $\underline{w}, \bar{w} \in$ $A C_{\text {loc }}\left(\left[T_{0}, T^{*}\right), \mathbb{R}\right)$ which satisfy, respectively,

$$
\begin{array}{r}
\underline{w}^{\prime} \leq G(t, \underline{w})+b(t), \quad \bar{w}^{\prime} \geq G(t, \bar{w})+b(t) \\
\text { a.e. in }\left[T_{0}, T^{*}\right) .
\end{array}
$$

We are interested in studying the following property:

$$
\begin{array}{r}
\underline{w}\left(T_{0}\right) \leq \bar{w}\left(T_{0}\right) \quad \text { implies } \underline{w}(t) \leq \bar{w}(t) \\
\forall t \in\left[\mathrm{T}_{0}, T^{*}\right) .
\end{array}
$$

In this way, we introduce the following definition. 
Definition 14. We say that comparison principle holds for (39) on an interval $\left[T_{0}, T^{*}\right), T \leq T_{0}<T^{*}$, if property (41) is fulfilled for all sub- and supersolutions $\underline{w}, \bar{w} \in$ $A C_{\mathrm{loc}}\left(\left[T_{0}, T^{*}\right), \mathbb{R}\right)$ of $(39)$ on $\left[T_{0}, T^{*}\right)$.

Now, we are able to state the next result.

Lemma 15. Let $T \leq T_{0}<T^{*}$. Let $G=G(t, u)$ and $b=b(t)$ be two arbitrary function. For any $M>0$, let there be a function $L=L(t)>0$ depending on $T_{0}, T^{*}, M$ such that

$$
\begin{gathered}
L(t)>0 \text { on }\left[T_{0}, T^{*}\right), L \in L^{1}\left(T_{0}, T^{*}\right), \\
\left|G\left(t, u_{1}\right)-G\left(t, u_{2}\right)\right| \leq L(t)\left|u_{1}-u_{2}\right| \\
\forall t \in\left[T_{0}, T^{*}\right), u_{1}, u_{2} \in[-M, M] .
\end{gathered}
$$

Then comparison principle (41) holds for (39) on an interval $\left[T_{0}, T^{*}\right), T \leq T_{0}<T^{*}$.

Proof. It is enough to use the idea of the proof of [14, Lemma 4.1]. Hence, this proof is left to the reader.

Corollary 16. Let $\lambda>0$, and let $g_{0}: \mathbb{R}_{+} \rightarrow \mathbb{R}_{+}$be a locally Lipschitz function on $\mathbb{R}_{+}$. Let $K$ be an arbitrary real number and $q(t)$ an arbitrary function. Let $T \leq T_{0}<T^{*}$. If $r(t)>0$ on $\left[T_{0}, T^{*}\right)$ and $r^{-1+\gamma} \in L^{1}\left(T_{0}, T^{*}\right)$, then comparison principle (41) holds for the Riccati differential equation

$$
w^{\prime}=\frac{1}{(\lambda r(t))^{\gamma-1}} g_{0}(|w|)+K \lambda q(t), \quad \text { a.e. in }\left[T_{0}, T^{*}\right) \text {. }
$$

Proof. It is clear that (43) is a particular case of (39) in particular for

$$
G(t, u)=\frac{1}{(\lambda r(t))^{\gamma-1}} g_{0}(|u|), \quad b(t)=K \lambda q(t) .
$$

Since $g_{0}$ is a locally Lipschitz function on $\mathbb{R}_{+}$, for every $M>0$ there is an $L_{0}>0$ depending on $M$ such that

$$
\left|g_{0}\left(s_{1}\right)-g_{0}\left(s_{2}\right)\right| \leq L_{0}\left|s_{1}-s_{2}\right|, \quad \forall s_{1}, s_{1} \in[-M, M] \text {. }
$$

Hence, for any $M>0$ and all $u_{1}, u_{2} \in[-M, M]$, we obtain:

$$
\begin{aligned}
\left|G\left(t, u_{1}\right)-G\left(t, u_{2}\right)\right| & =\frac{1}{(\lambda r(t))^{\gamma-1}}\left|g_{0}\left(\left|u_{1}\right|\right)-g_{0}\left(\left|u_{2}\right|\right)\right| \\
& \leq \frac{L_{0}}{(\lambda r(t))^{\gamma-1}}|| u_{1}|-| u_{2}|| \\
& \leq \frac{L_{0}}{(\lambda r(t))^{\gamma-1}}\left|u_{1}-u_{2}\right| .
\end{aligned}
$$

Thus, according to assumption $r^{-1+\gamma} \in L^{1}\left(T_{0}, T^{*}\right)$, the required condition (42) is fulfilled in particular for $L(t)=$ $L_{0}(\lambda r(t))^{-\gamma+1}$ and so Lemma 15 proves this corollary.
Before we give the proof of Theorem 3, we state and prove the next two propositions. In the first one, by a nonoscillatory solution $x(t)$ of the main equation (1), we get the existence of a supersolution $\bar{w}(t)$ of the Riccati differential equation (43) on the interval $\left(a_{1}, b_{1}\right)$ or $\left(a_{2}, b_{2}\right)$. In the second one, we construct two subsolutions $\underline{w}_{1}(t)$ and $\underline{w}_{2}(t)$ of (43) which blow up on intervals $\left(a_{1}, T_{1}^{*}\right) \subseteq\left(a_{1}, b_{1}\right)$ and $\left(a_{2}, T_{2}^{*}\right) \subseteq\left(a_{2}, b_{2}\right)$, respectively.

Proposition 17. Let $\Phi(u, v)$ and $f(u)$ satisfy (11), (12), and (16), respectively, and let $e(t)$ satisfy (2). Let $r(t)>0, q(t) \geq$ 0 and $q(t) \not \equiv 0$ on $\left[a_{1}, b_{1}\right] \cup\left[a_{2}, b_{2}\right]$. Let $x=x(t)$ be $a$ nonoscillatory solution of (1) and let, for some $T \geq t_{0}$ and $\lambda>0$, the function $\bar{w}=\bar{w}(t)$ be defined by

$$
\bar{w}(t)=-\frac{\lambda r(t) \Phi\left(x(t), x^{\prime}(t)\right)}{x(t)}, \quad t \geq T
$$

Then $\bar{w} \in A C_{\mathrm{loc}}([T, \infty), \mathbb{R})$ and $\bar{w}(t)$ satisfies the inequality

$$
\bar{w}^{\prime} \geq(\lambda r(t))^{1-\gamma} g_{0}(|\bar{w}|)+K \lambda q(t) \quad \text { a.e. in } J
$$

where $J=\left(a_{1}, b_{1}\right)$ if $x(t)<0$ and $J=\left(a_{2}, b_{2}\right)$ if $x(t)>0$.

Proof. Since $x=x(t)$ is a nonoscillatory solution of (1), there is a $T \geq t_{0}$ such that $x(t) \neq 0$ on $[T, \infty)$. Hence, $\bar{w}(t)$ is well defined by (47).

Because of (2), we have $e(t) / x(t) \leq 0$ for all $t \in J$, where $J=\left(a_{1}, b_{1}\right)$ if $x(t)<0$ and $J=\left(a_{2}, b_{2}\right)$ if $x(t)>0$ and since $\lambda>0$ we have

$$
-\lambda \frac{e(t)}{x(t)} \geq 0 \text { in } J
$$

Next, since $x$ and $r(t) \Phi\left(x(t), x^{\prime}(t)\right)$ are from $A C_{\text {loc }}\left(\left[t_{0}, \infty\right), \mathbb{R}\right)$, we can take the first derivative of $\bar{w}(t)$ for almost everywhere in $J$, which together with $r(t)>0$, $q(t) \geq 0$ and $q(t) \neq \equiv$ on $J$ gives

$$
\begin{aligned}
\bar{w}^{\prime}(t)= & -\frac{\lambda\left(r(t) \Phi\left(x(t), x^{\prime}(t)\right)\right)^{\prime}}{x(t)} \\
& +\frac{\lambda r(t) \Phi\left(x(t), x^{\prime}(t)\right)}{x^{2}(t)} x^{\prime}(t) \\
= & \frac{\lambda r(t)}{|x(t)|^{\gamma}} \Phi\left(x(t), x^{\prime}(t)\right) x^{\prime}(t)|x(t)|^{\gamma-2} \\
& +\lambda q(t) \frac{f(x(t))}{x(t)}-\lambda \frac{e(t)}{x(t)}
\end{aligned}
$$




$$
\begin{aligned}
& \geq \frac{\lambda r(t)}{|x(t)|^{\gamma}} g\left(\left|\Phi\left(x(t), x^{\prime}(t)\right)\right|\right)+K \lambda q(t)-\lambda \frac{e(t)}{x(t)} \\
& =\frac{\lambda r(t)}{|x(t)|^{\gamma}} g\left(\frac{|x(t)|}{\lambda r(t)}|\bar{w}(t)|\right)+K \lambda q(t)-\lambda \frac{e(t)}{x(t)} \\
& \geq \frac{\lambda r(t)}{|x(t)|^{\gamma}} \frac{|x(t)|^{\gamma}}{(\lambda r(t))^{\gamma}} g_{0}(|\bar{w}(t)|) \\
& \quad+K \lambda q(t)-\lambda \frac{e(t)}{x(t)} \quad \text { a.e. in } J,
\end{aligned}
$$

that is,

$$
\bar{w}^{\prime} \geq(\lambda r(t))^{1-\gamma} g_{0}(|\bar{w}|)+K \lambda q(t)-\lambda \frac{e(t)}{x(t)} \quad \text { a.e. in } J .
$$

Hence, (49) and (51) prove the desired inequality (48).

Proposition 18. Let (19) be satisfied. Let $R_{1}$ and $R_{2}$ be two arbitrary real numbers. Then there are two points $T_{1}^{*} \in\left(a_{1}, b_{1}\right)$ and $T_{2}^{*} \in\left(a_{2}, b_{2}\right)$ and two continuous functions $\underline{w}_{1}(t)$ and $\underline{w}_{2}(t)$, such that

$$
\begin{gathered}
\underline{w}_{i}\left(a_{i}\right) \leq R_{i}, \quad \lim _{t \rightarrow T_{i}^{*}} \underline{w}_{i}(t)=\infty, i \in\{1,2\}, \\
\underline{w}_{i}^{\prime} \leq(\lambda r(t))^{1-\gamma} g_{0}\left(\left|\underline{w}_{i}\right|\right)+K \lambda q(t) \\
\text { for a.e. } t \in\left(a_{i}, T_{i}^{*}\right), i \in\{1,2\} .
\end{gathered}
$$

Proof. Since the function $y(s)=\tan (s)$ is a bijection from the interval $(-\pi / 2, \pi / 2)$ into $\mathbb{R}$, we observe that there are two $s_{1}, s_{2} \in(-\pi / 2, \pi / 2)$ such that

$$
\tan \left(s_{i}\right) \leq R_{i}, \quad i \in\{1,2\} .
$$

Next, we define two functions $V_{1}(t)$ and $V_{2}(t)$ by

$$
V_{i}(t)=s_{i}+\frac{\pi}{c_{i}} \int_{a_{i}}^{t} C(\tau) d \tau, \quad t \in\left[a_{i}, b_{i}\right], i \in\{1,2\},
$$

where the real numbers $c_{1}, c_{2}$ and the function $C(t)$ are defined in (19). From (19) and (54), one can immediately conclude that

$$
V_{i}\left(a_{i}\right)=s_{i}<\frac{\pi}{2}, \quad V_{i}\left(b_{i}\right)=s_{i}+\pi>\frac{\pi}{2}, i \in\{1,2\} .
$$

Since $C \in L_{\text {loc }}^{1}\left(\left(t_{0}, \infty\right), \mathbb{R}\right)$, it follows that $C \in L^{1}\left(\left(t_{0}, \infty\right), \mathbb{R}\right)$, which implies that $V_{i} \in A C\left(\left[a_{i}, b_{i}\right], \mathbb{R}\right), i \in\{1,2\}$. Therefore, exploiting the fact that in (55) we have $V_{i} \in C\left(\left[a_{i}, b_{i}\right], \mathbb{R}\right)$, we obtain the existence of two points $T_{1}^{*} \in\left(a_{1}, b_{1}\right)$ and $T_{2}^{*} \in$ $\left(a_{2}, b_{2}\right)$ such that

$$
\begin{array}{r}
V_{i}\left(T_{i}^{*}\right)=\frac{\pi}{2}, \quad-\frac{\pi}{2}<V_{i}(t)<\frac{\pi}{2} \\
\forall t \in\left[a_{i}, T_{i}^{*}\right), i \in\{1,2\} .
\end{array}
$$

Now, we are able to define the following two functions $\underline{\omega}_{1}(t)$ and $\underline{\omega}_{2}(t)$ by

$$
\underline{\omega}_{i}(t)=\tan \left(V_{\mathrm{i}}(t)\right), \quad t \in\left[a_{i}, T_{i}^{*}\right), i \in\{1,2\} .
$$

That are well defined because of (56). Moreover, from (53), (54), and (56) we obtain

$$
\begin{gathered}
\underline{\omega}_{i}\left(a_{i}\right)=\tan \left(s_{i}\right) \leq R_{i}, \\
\lim _{t \rightarrow T_{m}^{*}} \underline{\omega}_{i}(t)=\tan \left(V_{i}\left(T_{i}^{*}\right)\right)=\tan \left(\frac{\pi}{2}\right)=\infty .
\end{gathered}
$$

Also, since $V_{i} \in A C\left(\left[a_{i}, b_{i}\right], \mathbb{R}\right)$, we can take the first derivative of $\underline{\omega}_{i}(t)$ and hence from (19) and (57) we obtain

$$
\begin{aligned}
\underline{w}_{i}^{\prime}(t) & =\frac{\pi}{c_{i}} C(t) \frac{1}{\cos ^{2}\left(V_{i}(t)\right)} \\
& =\frac{\pi}{c_{i}} C(t)\left(\left|\underline{\omega}_{i}(t)\right|^{2}+1\right) \\
& \leq \frac{\pi}{c_{i}} C(t)\left[g_{0}\left(\left|\underline{\omega}_{i}(t)\right|\right)+M_{0}+1\right] \\
& \leq \frac{\pi}{c_{i}} C(t) g_{0}\left(\left|\underline{\omega}_{i}(t)\right|\right)+\frac{\pi}{c_{i}} C(t)\left(M_{0}+1\right) \\
& \leq(\lambda r(t))^{1-\gamma} g_{0}\left(\left|\underline{\omega}_{i}(t)\right|\right)+K \lambda q(t), \quad \text { a.e. in }\left(a_{i}, T_{i}^{*}\right),
\end{aligned}
$$

which together with (58) proves this proposition.

Now we are able to present the proof of Theorem 3 based on Lemma 15 and Propositions 17 and 18.

Proof of Theorem 3. Assuming the contrary, then there is a nonoscillatory solution $x(t)$ and $T \geq t_{0}$ such that $x(t) \neq 0$ for all $t \geq T, x(t)$ and $r(t) \Phi\left(x, x^{\prime}\right)$ are from $A C_{\mathrm{loc}}\left(\left[t_{0}, \infty\right), \mathbb{R}\right)$. In order to simplify notation, let every $j \in \mathbb{N}$ be fixed and omitted in the notation. For instance, instead of $\left(a_{1 j}, b_{1 j}\right)$ and $\left(a_{2 j}, b_{2 j}\right)$ we write $\left(a_{1}, b_{1}\right)$ and $\left(a_{2}, b_{2}\right)$, respectively, and so on.

On one hand, we observe by Proposition 17 that the function $\bar{w}(t)$ defined by (47) is a supersolution of the following Riccati differential equation:

$$
w^{\prime}=(\lambda r(t))^{1-\gamma} g_{0}(|w|)+K \lambda q(t) \quad \text { a.e. in } J,
$$

where $\bar{w} \in A C_{\mathrm{loc}}(J, \mathbb{R})$ and $J=\left(a_{1}, b_{1}\right)$ if $x(t)<0$ and $J=$ $\left(a_{2}, b_{2}\right)$ if $x(t)>0$; see the proof of Proposition 17. Let for instance $x(t)<0$ and thus $J=\left(a_{1}, b_{1}\right)$.

On the other hand, by Proposition 18 there are a number $T_{1}^{*} \in\left(a_{1}, b_{1}\right)$ and subsolutions $\underline{w}_{1}(t), \underline{w}_{1} \in A C\left(\left[a_{1}, T_{1}^{*}\right), \mathbb{R}\right)$ of the Riccati differential equation (60) such that

$$
\underline{w}_{1}\left(a_{1}\right) \leq \bar{w}\left(a_{1}\right), \quad \lim _{t \rightarrow T_{1}^{*}} \underline{w}_{1}(t)=\infty,
$$

(in the case when $J=\left(a_{2}, b_{2}\right)$, then we work with $T_{2}^{*} \in\left(a_{2}, b_{2}\right)$ and the other subsolution $\left.\underline{w}_{2}(t), \underline{w}_{2} \in A C\left(\left[a_{2}, T_{2}^{*}\right), \mathbb{R}\right)\right)$. Hence by Corollary 16 and $(61)$ we conclude that $\underline{w}_{1}(t) \leq \bar{w}(t)$ for all $t \in\left[a_{1}, T_{1}^{*}\right)$ and therefore,

$$
\infty=\lim _{t \rightarrow T_{1}^{*}} \underline{w}_{1}(t) \leq \lim _{t \rightarrow T_{1}^{*}} \bar{w}(t),
$$

which contradicts $\bar{w} \in A C_{\mathrm{loc}}([T, \infty), \mathbb{R})$. Thus, the assumption that $x(t)$ is nonoscillatory is not possible and hence every solution of (1) is oscillatory. 
Proof of Theorem 4. There is only one difference between Theorems 3 and 4, and it is the difference between conditions (19) and (20). Hence, Theorem 4 immediately follows from Theorem 3 provided that we prove the equivalence between (19) and (20).

First of all, we need the following two lemmas.

Lemma 19. Let $a, b$, and $c$ be positive real numbers, and $\gamma>1$. Then the existence of a positive real number $\lambda$ such that

$$
c \leq \min \left\{\frac{a}{\lambda^{\gamma-1}}, b \lambda\right\}
$$

is equivalent to

$$
c \leq a^{1 / \gamma} b^{1 / \gamma^{\prime}}
$$

Here $\gamma^{\prime}:=\gamma /(\gamma-1)$ is the conjugate exponent of $\gamma$.

Proof. Let us define $g(\lambda)=\min \left\{a / \lambda^{\gamma-1}, b \lambda\right\}$. Since $\lambda \mapsto$ $a / \lambda^{\gamma-1}$ is decreasing and $\lambda \mapsto b \lambda$ is increasing, there exists a unique point of maximum of $g$. It is achieved at $\lambda=\lambda_{0}$ which is a solution of $a / \lambda_{0}^{\gamma-1}=b \lambda_{0}$, hence $\lambda_{0}=(a / b)^{1 / \gamma}$.

If there is a positive real number $\lambda$ such that $c \leq g(\lambda)$, then $c \leq g\left(\lambda_{0}\right)=b \lambda_{0}=b(a / b)^{1 / \gamma}=a^{1 / \gamma} b^{1 / \gamma^{\prime}}$. Conversely, if $c \leq a^{1 / \gamma} b^{1 / \gamma^{\prime}}$, then $c \leq g\left(\lambda_{0}\right)$, and the equivalence in the lemma is proved.

The preceding lemma is a special case of the following more general statement.

Lemma 20. Assume that $a(t), b(t)$, and $c(t)$ are positive real functions defined on a subset $J \subseteq \mathbb{R}$. Then the existence of a positive real number $\lambda$ such that for all $t \in J$,

$$
c(t) \leq \min \left\{\frac{a(t)}{\lambda^{\gamma-1}}, b(t) \lambda\right\},
$$

is equivalent to

$$
\sup _{t \in J} \frac{c(t)}{b(t)} \leq\left(\inf _{t \in J} \frac{a(t)}{c(t)}\right)^{1 /(\gamma-1)} .
$$

Proof. The condition that there exists $\lambda>0$ such that (65) is true for all $t \in J$ is equivalent with the following two inequalities, which have to hold simultaneously:

$$
c(t) \leq \frac{a(t)}{\lambda^{\gamma-1}}, \quad c(t) \leq b(t) \lambda
$$

that is, with

$$
\frac{c(t)}{b(t)} \leq \lambda \leq\left(\frac{a(t)}{c(t)}\right)^{1 /(\gamma-1)} \quad \forall t \in J .
$$

Taking the supremum of the left-hand side and the infimum of the right-hand side, we obtain (66). Conversely, if (66) is satisfied, then, since the left-hand side in (66) cannot be equal to zero, while the right-hand side is less than $\infty$, then inequality (66) defines a nonempty closed interval in $\mathbb{R}$ (possibly reducing to just one point), in which we can choose any positive $\lambda$. Hence, (67) is satisfied for all $t \in J$, and therefore (65) as well.
Remark 21. If $a=a(t), b=b(t)$, and $c=c(t)$, appearing in Lemma 20, are constant functions, then condition (66) is equivalent to $c / b=(a / c)^{1 /(\gamma-1)}$, that is, to (64).

Proof of Equivalence of (19) and (20). We will use Lemma 20. Let us fix $J_{i j}$, where $i \in\{1,2\}$ and $j \in \mathbb{N}$. We see that that the inequality appearing in the second line of condition (19) is of the form (65), where

$$
\begin{gathered}
c(t):=\frac{1}{c_{i j}} C(t), \quad a(t):=\frac{1}{\pi} r(t)^{1-\gamma}, \\
b(t):=\frac{K q(t)}{\pi\left(M_{0}+1\right)} .
\end{gathered}
$$

Condition (66) is equivalent to

$$
\begin{aligned}
\sup _{t \in J_{i j}} \frac{C(t)}{q(t)} & \leq \frac{K c_{i j}^{\gamma^{\prime}}}{\pi^{\gamma^{\prime}}\left(M_{0}+1\right)} \inf _{t \in J_{i j}} \frac{1}{r(t) C(t)^{\gamma-1}} \\
& =\frac{K c_{i j}^{\gamma^{\prime}}}{\left(M_{0}+1\right) \sup _{t \in J_{i j}} r(t) C(t)^{\gamma-1}}\left(\frac{c_{i j}}{\pi}\right)^{\gamma^{\prime}} .
\end{aligned}
$$

This inequality is equivalent to the corresponding one in (19), and the claim follows from Lemma 19.

Proof of Corollary 6. Substituting $\inf _{t \in J_{i j}} q(t)$ instead of $q(t)$, and $\sup _{t \in J_{i j}} r(t)$ instead of $r(t)$ in the inequality appearing in the second line of (20), after a short computation we obtain a stronger inequality than (20) as

$$
\begin{aligned}
& \frac{1}{c_{i j}} \sup _{t \in J_{i j}} C(t) \\
& \quad \leq \frac{1}{\pi}\left(\frac{\operatorname{Kinf}_{t \in J_{i j}} q(t)}{\left(M_{0}+1\right) \sup _{t \in J_{i j}} r(t)}\right)^{(\gamma-1) / \gamma} \quad \forall i \in\{1,2\}, j \in \mathbb{N} .
\end{aligned}
$$

Substituting $C(t) \equiv 1$ we obtain that the left-hand side of (71) is equal to $\left|J_{i j}\right|^{-1}$, and the resulting inequality is equivalent to (21). Since (21) implies (20), the claim is proved.

Remark 22. Here we show that the choice of $C(t) \equiv 1$ in the proof of Corollary 6 is the best possible. To prove this, note that on the left-hand side of (71) we have the expression depending on an auxiliary function $C(t)$, and this function is absent on the right-hand side. Therefore, it has sense to try to find a function $C(t), t \in J_{i j}$, such that the value of $Q(C):=\left(1 / c_{i j}\right) \sup _{t \in J_{i j}} C(t)$ is minimal (note that $c_{i j}$ depends on the function $C=C(t)$ as well). It is easy to see that the minimum is achieved for $C(t) \equiv 1$ (or any positive constant). Indeed, since $Q(C)=Q(\mu c)$ for any $\mu>0$, by taking $\mu=$ $\left(\sup _{t \in J_{i j}} C(t)\right)^{-1}$ it suffices to assume that $\sup _{t \in J_{i j}} C(t)=1$. The value of $Q(C)$ is minimal if $c_{i j}=\int_{J_{i j}} C(\tau) d \tau$ is maximal possible, and since $0 \leq C(t) \leq 1$, it is clear that the maximum of $c_{i j}=c_{i j}(C)$ is achieved when $C(t) \equiv 1$. 
Proof of Corollary 7. It is clear that the condition (23) implies (21). Furthermore, since the lengths $\left|J_{i j}\right|$ are uniformly bounded from below by a positive constant, see (23), then obviously $a_{1 j} \rightarrow \infty$ as $j \rightarrow \infty$. Thus, the claim follows immediately from Corollary 6.

Proof of Corollary 10. Let $C(t):=q(t)$. Then the required condition (19) is clearly satisfied because of assumption (30). Hence, this corollary immediately follows from Theorem 3.

\section{An Extension of Condition (12) to the Case of $\gamma>1$}

In this section, we consider the oscillation of (1) in the case when $\gamma \in(1,2)$ is allowed in the assumption (12). In this way, the assumption (11) is slightly modified by a real number $p>$ 1 such that

$$
|u|^{(p-1) \gamma-p} v \Phi(u, v) \geq g(|\Phi(u, v)|)
$$

where (12) is supposed, that is, $g(c s) \geq c^{\gamma} g_{0}(s)$ for every $c, s>$ 0 and for a locally Lipschitz function $g_{0}:[0, \infty) \rightarrow[0, \infty)$ for which there exists a constant $M_{0}$ such that $g_{0}(s)+M_{0} \geq s^{p}$ for all $s \in[0, \infty)$.

For the function $f$, we assume that there exists a constant $K>0$ such that

$$
f(u) \operatorname{sign}(u) \geq K|u|^{p-1} \quad \text { for every } u \in \mathbb{R} .
$$

We will need the following two lemmas.

Lemma 23. Let $a<b$, and let $f:[a, b] \rightarrow[0, \infty)$ be a measurable function. Then,

$$
\int_{a}^{b} f(t) d t \geq 1
$$

if and only if there exists a function $g \in L_{1}([a, b],[0, \infty))$, $\|g\|_{1}=1$, such that

$$
f(t) \geq g(t), \quad \forall t \in[a, b] .
$$

Proof. We assume (74). If $\int_{a}^{b} f(t) d t$ is finite, we may choose $g(x):=f(x) /\|f\|_{1}$.

For $\int_{a}^{b} f(t) d t=\infty$, we may define for every natural number $n$ a function

$$
f_{n}(t)= \begin{cases}f(t), & f(t) \leq n, \\ n, & f(t)>n .\end{cases}
$$

Since $f_{n}(t) \leq n$, we have $f_{n} \in L_{1}([a, b], \mathbb{R})$. Obviously, $\lim _{n} \int_{a}^{b} f_{n}(t) d t$ is also infinite. This implies that there exists an $n_{0}$ such that $\int_{a}^{b} f_{n_{0}}(t) d t \geq 1$. We define $g(t):=f_{n_{0}}(t) /\left\|f_{n_{0}}\right\|_{1}$.

Now, we assume (75). Integrating over the whole interval, we get

$$
\int_{a}^{b} f(t) d t \geq \int_{a}^{b} g(t) d t=1
$$

Lemma 24. Let $p>1$. There exists a unique function $y=y(s)$ that satisfies the following Cauchy problem:

$$
\begin{gathered}
y^{\prime}(s)=1+|y(s)|^{p}, \quad s \in \mathbb{R}, \\
y(0)=0 .
\end{gathered}
$$

Furthermore, there exists a finite real number $S^{*}$ such that

$$
\lim _{s \rightarrow \pm S^{*}} y(s)= \pm \infty
$$

and $y \in C^{1}\left(\left(-S^{*}, S^{*}\right), \mathbb{R}\right)$ is injective and monotonous. We have $S^{*}=(\pi / p)(1 /(\sin (\pi / p)))$ and $y$ is odd.

We will denote by $\tan _{p}$ the solution of (78)-(79).

Proof. Obviously every solution to (78) is injective and monotonous on a connected set, since $y^{\prime}=1+|y|^{p} \geq 1>0$.

Let $f(x)=1 /\left(1+|x|^{p}\right)$. Obviously $1 \geq f(x)>0$ for all $x \in \mathbb{R}$ and $C_{p}>\left|f^{\prime}(x)\right|>0$ for some constants $C_{p} \in \mathbb{R}$. We may assume $C_{p}>1$. Hence, $z^{\prime}=f(t), z(0)=0$ has (by $\left[17\right.$, Theorem 3.1]) a locally unique solution on $\left(-1 / C_{p}, 1 / C_{p}\right)$. By the same argument $z$ can be extended to the whole of $\mathbb{R}$, interval by interval of type $\left((n-1) / C_{p},(n+1) / C_{p}\right)$ for all $n \in \mathbb{Z}$.

We know from [18] that the for the integral of the lefthand side we have

$$
\int_{0}^{\infty} f(x) d x=\int_{0}^{\infty} \frac{d x}{1+x^{p}}=\frac{\pi}{p} \frac{1}{\sin (\pi / p)}=S^{*},
$$

so $\lim _{t \rightarrow \pm \infty} z(t)= \pm S^{*}$.

Since $z^{\prime}(t) \neq 0, z$ is injective, monotonous, and of class $C^{1}\left(\mathbb{R},\left(-S^{*}, S^{*}\right)\right)$ and so we define $y(s)=z^{-1}(s)$. It immediately follows that $y \in C^{1}\left(\left(-S^{*}, S^{*}\right), \mathbb{R}\right)$ and that $y$ is injective and monotonous. Also, by continuity of $z$, we have $\lim _{s \rightarrow \pm S^{*}} y(s)=\lim _{w \rightarrow \pm \infty} y(z(w))=\lim _{w \rightarrow \pm \infty} w= \pm \infty$.

Differentiating, it also follows that $y^{\prime}=1+|y|^{p}$ and by definition of $y, y(0)=z^{-1}(0)=0$. So, $y$ is a solution of (78)(79). Since any other such solution must have $z$ as its inverse, $y$ is unique on its domain.

Proposition 25. Let $a<b$ and $\gamma>p>1$. Assume (72) and (73) and let $q(t) \geq 0$ and $e(t) \leq 0$ (or $e(t) \geq 0$ ) for all $t \in[a, b]$. If there exists a constant $\lambda>0$ such that

$$
\frac{p}{2 \pi} \sin \left(\frac{\pi}{p}\right) \int_{a}^{b} \min \left\{\frac{p-1}{r^{\gamma-1}(t) \lambda^{\gamma-1}}, \frac{K \lambda q(t)}{1+M_{0}}\right\} d t \geq 1,
$$

then for any solution $x(t)$ of (1) with $x(a) \geq 0$ (or $x(a) \leq 0$ ) there exists a number $t^{*} \in[a, b]$ such that $x\left(t^{*}\right)=0$.

Proof. We assume that $x(t) \neq 0$ for all $t \in[a, b]$. Then we may define the function

$$
\bar{\omega}(t):=-\frac{\lambda r(t) \Phi\left(x(t), x^{\prime}(t)\right)}{\operatorname{sign}(x(t))|x(t)|^{p-1}} .
$$


This function is well defined on $[a, b]$ and its derivative is

$$
\begin{aligned}
\bar{w}^{\prime}(t)= & -\frac{\lambda\left(r(t) \Phi\left(x(t), x^{\prime}(t)\right)\right)^{\prime}}{\operatorname{sign}(x(t))|x(t)|^{p-1}} \\
& +\frac{(p-1) \lambda r(t) \Phi\left(x(t), x^{\prime}(t)\right)}{|x(t)|^{p}} x^{\prime}(t) .
\end{aligned}
$$

Using the fact that $x(t)$ is a solution of (1) and omitting $t$ from $x$ and $x^{\prime}$ for clarity, we get

$$
\begin{aligned}
& \bar{w}^{\prime}(t)=\frac{\lambda(q(t) f(x)-e(t))}{\operatorname{sign}(x)|x|^{p-1}}+\frac{(p-1) \lambda r(t) \Phi\left(x, x^{\prime}\right)}{x^{p}} x^{\prime} \\
& =\frac{(p-1) \lambda r(t) \Phi\left(x, x^{\prime}\right)}{|x|^{p}} x^{\prime}+\lambda q(t) \frac{f(x)}{\operatorname{sign}(x)|x|^{p-1}} \\
& -\lambda \frac{e(t)}{\operatorname{sign}(x)|x|^{p-1}} \\
& =\frac{(p-1) \lambda r(t)}{|x|^{\gamma(p-1)}} \Phi\left(x, x^{\prime}\right) x^{\prime}|x|^{(p-1) \gamma-p} \\
& +\lambda q(t) \frac{f(x)}{\operatorname{sign}(x)|x|^{p-1}}-\lambda \frac{e(t)}{\operatorname{sign}(x)|x|^{p-1}} \\
& \stackrel{(72)}{\geq} \frac{(p-1) \lambda r(t)}{|x|^{\gamma(p-1)}} g\left(\left|\Phi\left(x, x^{\prime}\right)\right|\right) \\
& +\lambda q(t) \frac{f(x)}{\operatorname{sign}(x)|x|^{p-1}} \\
& -\lambda \frac{e(t)}{\operatorname{sign}(x)|x|^{p-1}} \\
& =\frac{(p-1) \lambda r(t)}{|x|^{\gamma(p-1)}} g\left(\frac{|x|^{p-1}}{\lambda r(t)}|\bar{\omega}(t)|\right) \\
& +\lambda q(t) \frac{f(x)}{\operatorname{sign}(x)|x|^{p-1}}-\lambda \frac{e(t)}{\operatorname{sign}(x)|x|^{p-1}} \\
& \stackrel{(12)}{\geq} \frac{(p-1) \lambda r(t)}{|x|^{\gamma(p-1)}}\left(\frac{|x|^{p-1}}{\lambda r(t)}\right)^{\gamma} g_{0}(|\bar{\omega}(t)|) \\
& +\lambda q(t) \frac{f(x)}{\operatorname{sign}(x)|x|^{p-1}}-\lambda \frac{e(t)}{\operatorname{sign}(x)|x|^{p-1}} \\
& =\frac{p-1}{(\lambda r(t))^{\gamma-1}} g_{0}(|\bar{\omega}(t)|)+\lambda q(t) \frac{f(x)}{\operatorname{sign}(x)|x|^{p-1}} \\
& -\lambda \frac{e(t)}{\operatorname{sign}(x)|x|^{p-1}} \\
& \stackrel{(73)}{\geq} \frac{p-1}{(\lambda r(t))^{\gamma-1}} g_{0}(|\bar{\omega}(t)|)+\lambda q(t) K \\
& -\lambda \frac{e(t)}{\operatorname{sign}(x)|x|^{p-1}} .
\end{aligned}
$$

Since $e(t) /\left(\operatorname{sign}(x)|x|^{p-1}\right) \leq 0$, we have

$$
\bar{w}^{\prime}(t) \geq \frac{p-1}{(\lambda r(t))^{\gamma-1}} g_{0}(|\bar{\omega}(t)|)+\lambda K q(t) .
$$
that

We apply Lemma 23 to (82) to obtain a function $C(t)$ such

$$
\int_{a}^{b} C(t) d t=1
$$

and by (75)

$$
0 \leq C(t) \leq \frac{p}{2 \pi} \sin \left(\frac{\pi}{p}\right) \min \left\{\frac{p-1}{r^{\gamma-1}(t) \lambda^{\gamma-1}}, \frac{K \lambda q(t)}{1+M_{0}}\right\} .
$$

Let $s_{0} \in(-(\pi / p)(1 / \sin (\pi / p)),(\pi / p)(1 / \sin (\pi / p)))$ be such that $\tan _{p}\left(s_{0}\right) \leq \bar{\omega}(a)$. We define the function

$$
V(t)=s_{0}+\frac{2 \pi}{p} \frac{1}{\sin (\pi / p)} \int_{a}^{t} C(\tau) d \tau
$$

Note that $(-(\pi / p)(1 / \sin (\pi / p)))<s_{0}=V(a)<$ $(\pi / p)(1 / \sin (\pi / p))$ and $V(b)=s_{0}+(2 \pi / p)(1 / \sin (\pi / p))>$ $(\pi / p)(1 / \sin (\pi / p))$. Since $V$ is continuous, there exists a $T^{*} \epsilon$ $(a, b)$ such that $V(t)<(\pi / p)(1 / \sin (\pi / p))$ for $t \in\left[a, T^{*}\right)$ and $V\left(T^{*}\right)=(\pi / p)(1 / \sin (\pi / p))$.

We define

$$
\underline{\omega}(t):=\tan _{p}(V(t)), t \in\left[a, T^{*}\right),
$$

and note that by Lemma $24, \lim _{t \rightarrow T^{*}} \underline{\omega}(t)=+\infty$, where $\tan _{p}(s)=y(s)$ and $y(s)$ is determined by Lemma 24 .

The derivative of $\underline{\omega}$ then satisfies

$$
\begin{aligned}
\underline{\omega}^{\prime}(t) & =\tan _{p}^{\prime}(V(t)) V^{\prime}(t) \\
& =\left(1+\tan _{p}^{p}(V(t))\right) V^{\prime}(t) \\
& =\frac{2 \pi}{p} \frac{1}{\sin (\pi / p)}\left(1+\underline{\omega}^{p}(t)\right) C(t) \\
& \leq\left(1+M_{0}+g_{0}(|\underline{\omega}(t)|)\right) \min \left\{\frac{p-1}{r^{\gamma-1}(t) \lambda^{\gamma-1}}, \frac{K \lambda q(t)}{1+M_{0}}\right\} \\
& \leq \frac{p-1}{r^{\gamma-1}(t) \lambda^{\gamma-1}} g_{0}(|\underline{\omega}(t)|)+K \lambda q(t) .
\end{aligned}
$$

Thus, we may apply the comparison principle of Lemma 15 to $\underline{\omega}(a) \leq \bar{\omega}(a)$ and their respective differential inequalities to conclude that $\underline{\omega}(t) \leq \bar{\omega}(t)$ for all $t \in[a, b]$. But, since $\underline{\omega} \rightarrow+\infty$ as $t \rightarrow \bar{T}^{*}<b$ and $\bar{\omega}(t)$ is well defined on the whole segment $[a, b]$, we arrive at a contradiction. Hence, $x(t)>0$ cannot hold for all $t \in[a, b]$, and since $x(t)$ is continuous, there exists a $t^{*} \in[a, b]$ such that $x\left(t^{*}\right)=0$.

Theorem 26. Let $\gamma>p>1$. Assume (72) and (73) and let for any $T>t_{0}$ there exist $T \leq a_{1}<b_{1} \leq a_{2}<b_{2}$ such that $g(t) \geq 0$ on $\left[a_{1}, b_{1}\right] \cup\left[a_{2}, b_{2}\right]$ and $e(t) \leq 0$ on $\left[a_{1}, b_{1}\right]$ and $e(t) \geq 0$ on 
$\left[a_{2}, b_{2}\right]$. If for both $i \in\{1,2\}$ there exist constants $\lambda_{i}>0$ such that

$$
\frac{p}{2 \pi} \sin (\pi / p) \int_{a_{i}}^{b_{i}} \min \left\{\frac{p-1}{r^{\gamma-1}(t) \lambda_{i}^{\gamma-1}}, \frac{K \lambda_{i} q(t)}{1+M_{0}}\right\} d t \geq 1,
$$

then (1) is oscillatory.

Proof. For (1) to be oscillatory it is sufficient that for every $n \in \mathbb{N}$ there exists a $t_{n}>n$ such that $x\left(t_{n}\right)=0$.

Let $n \in \mathbb{N}$ and let $n<a_{1}<b_{1} \leq a_{2}<b_{2}$ as assumed in the theorem. It is enough to show that every solution $x(t)$ of (1) has a zero on $\left[a_{1}, b_{2}\right]$. If $x\left(a_{1}\right) \geq 0$, we apply Proposition 25 to the segment $\left[a_{1}, b_{1}\right]$ to obtain the sought zero. If $x\left(a_{2}\right) \leq 0$ we again apply Proposition 25 to the segment $\left[a_{2}, b_{2}\right]$. Suppose $x\left(a_{1}\right)<0$ and $x\left(a_{2}\right)>0$. Then, since $x$ is continuous, there exists a number $t_{1} \in\left[a_{1}, a_{2}\right]$ such that $x\left(t_{1}\right)=0$.

\section{Acknowledgment}

This work is supported by the Ministry of Science of the Republic of Croatia under Grant no. 036-0361621-1291.

\section{References}

[1] Q. Kong, "Nonoscillation and oscillation of second order halflinear differential equations," Journal of Mathematical Analysis and Applications, vol. 332, no. 1, pp. 512-522, 2007.

[2] Q.-R. Wang and Q.-G. Yang, "Interval criteria for oscillation of second-order half-linear differential equations," Journal of Mathematical Analysis and Applications, vol. 291, no. 1, pp. 224236, 2004.

[3] Q. Long and Q.-R. Wang, "New oscillation criteria of secondorder nonlinear differential equations," Applied Mathematics and Computation, vol. 212, no. 2, pp. 357-365, 2009.

[4] Z. Zheng and F. Meng, "Oscillation criteria for forced secondorder quasi-linear differential equations," Mathematical and Computer Modelling, vol. 45, no. 1-2, pp. 215-220, 2007.

[5] D. Çakmak and A. Tiryaki, "Oscillation criteria for certain forced second-order nonlinear differential equations," Applied Mathematics Letters, vol. 17, no. 3, pp. 275-279, 2004.

[6] F. Jiang and F. Meng, "New oscillation criteria for a class of second-order nonlinear forced differential equations," Journal of Mathematical Analysis and Applications, vol. 336, no. 2, pp. 1476-1485, 2007.

[7] Q. Yang, "Interval oscillation criteria for a forced second order nonlinear ordinary differential equations with oscillatory potential," Applied Mathematics and Computation, vol. 135, no. 1, pp. 49-64, 2003.

[8] A. Tiryaki and B. Ayanlar, "Oscillation theorems for certain nonlinear differential equations of second order," Computers \& Mathematics with Applications, vol. 47, no. 1, pp. 149-159, 2004.

[9] J. Jaroš, T. Kusano, and N. Yoshida, "Generalized Picone's formula and forced oscillations in quasilinear differential equations of the second order," Archivum Mathematicum, vol. 38, no. 1, pp. 53-59, 2002.

[10] R. P. Agarwal, S. R. Grace, and D. O'Regan, Oscillation Theory for Second Order Linear, Half-Linear, Superlinear and Sublinear Dynamic Equations, Kluwer Academic Publishers, Dordrecht, The Netherlands, 2002.
[11] S. Jing, "A new oscillation criterion for forced second-order quasilinear differential equations," Discrete Dynamics in Nature and Society, vol. 2011, Article ID 428976, 8 pages, 2011.

[12] W.-T. Li and S. S. Cheng, "An oscillation criterion for nonhomogeneous half-linear differential equations," Applied Mathematics Letters, vol. 15, no. 3, pp. 259-263, 2002.

[13] J. S. W. Wong, "Oscillation criteria for a forced second-order linear differential equation," Journal of Mathematical Analysis and Applications, vol. 231, no. 1, pp. 235-240, 1999.

[14] M. Pašić, "Fite-Wintner-Leighton type oscillation criteria for second-order differential equations with nonlinear damping," Abstract and Applied Analysis, vol. 2013, Article ID 852180, 10 pages, 2013.

[15] M. Pašić, "New interval oscillation criteria for forced secondorder differential equations with nonlinear damping," International Journal of Mathematical Analysis, vol. 7, no. 25, pp. 12391255, 2013.

[16] L. Gasiński and N. S. Papageorgiou, Nonsmooth Critical Point Theory and Nonlinear Boundary Value Problems, vol. 8 of Series in Mathematical Analysis and Applications, Edited by R. P. Agarwal and D. O'Regan, Chapman \& Hall/CRC, Boca Raton, Fla, USA, 2005.

[17] S. Lang, Real and Functional Analysis, vol. 142 of Graduate Texts in Mathematics, Springer-Verlag, New York, NY, USA, 3rd edition, 1993.

[18] I. N. Bronshtein, K. A. Semendyayev, G. Musiol, and H. Muehlig, Handbook of Mathematics, Springer, Berlin, Germany, 5th edition, 2007. 


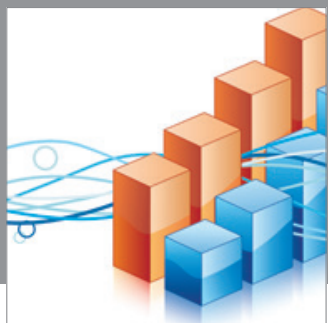

Advances in

Operations Research

mansans

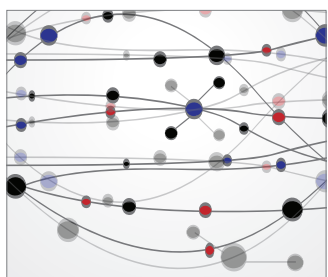

The Scientific World Journal
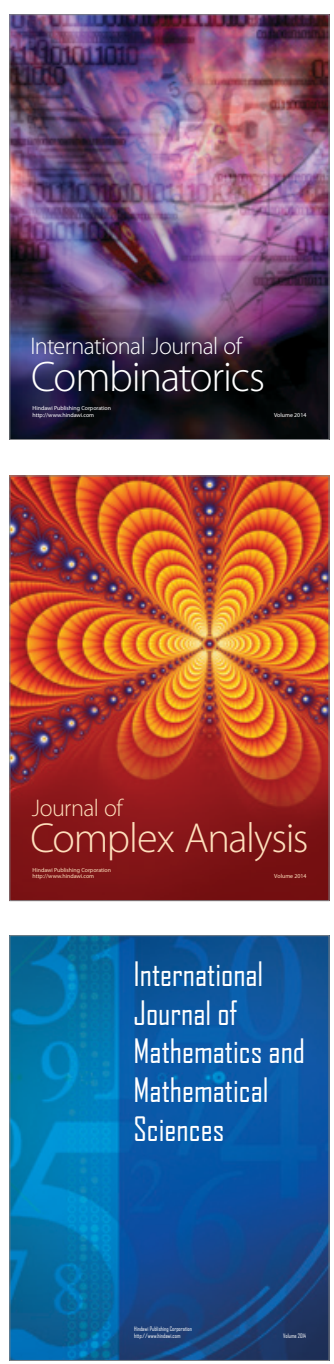
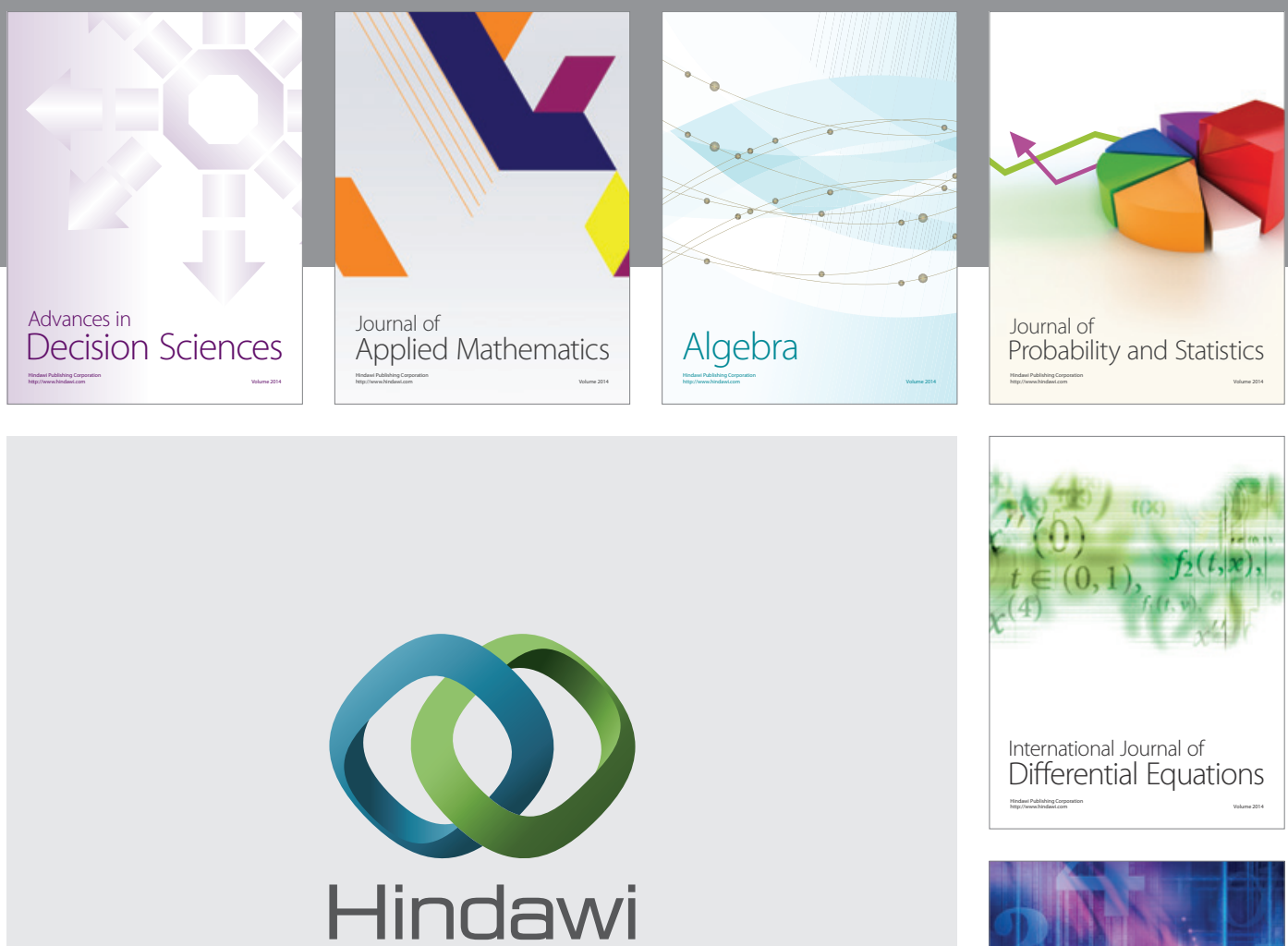

Submit your manuscripts at http://www.hindawi.com
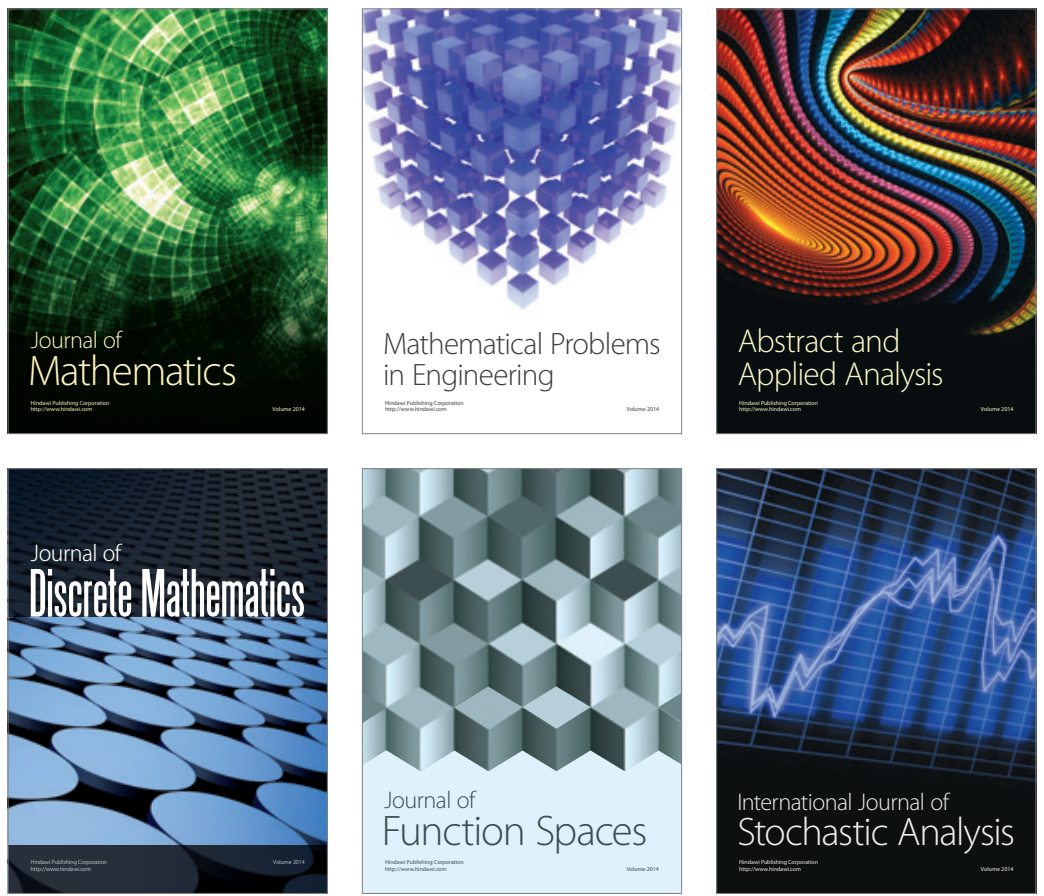

Journal of

Function Spaces

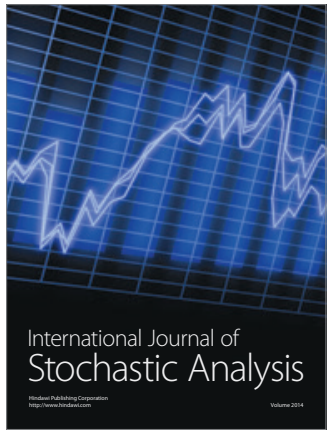

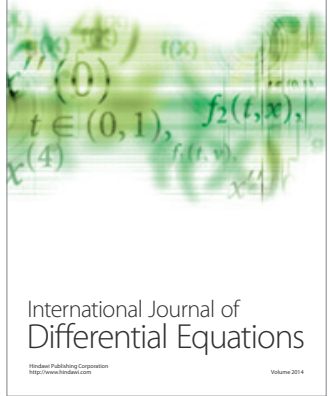
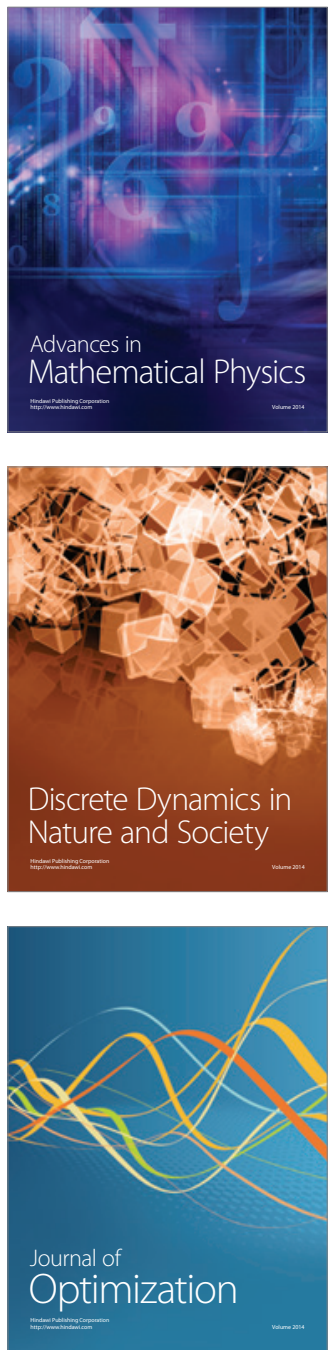